INRA Prod. Anim., 2017, 30 (2), $165-178$

\section{Aménités et impacts sur l'environnement des exploitations françaises élevant des bovins pour la viande}

M. DOREAU, A. FARRUGGIA, P. VEYSSET

UMR1213 Herbivores, INRA, Vetagro Sup, 63122, Saint-Genès-Champanelle, France

Courriel : anne.farruggia@inra.fr

\begin{abstract}
Reconnues pour leurs aménités environnementales, les exploitations élevant des bovins pour la viande font aussi l'objet de critiques virulentes sur les impacts environnementaux qu'elles engendrent. Quel bilan environnemental peut-on faire aujourd'hui de cet élevage ?
\end{abstract}

Les aménités environnementales liées aux exploitations françaises élevant des bovins pour la viande (que l'on appellera dans la suite du texte bovins viande) sont souvent mises en avant, en particulier par rapport à la valorisation des espaces peu productifs, la production de protéines à partir de ressources fourragères non compétitrices avec l'alimentation humaine, le stockage de carbone par les sols, l'utilisation de pesticides et la biodiversité. Dans le même temps, les pressions environnementales exercées par cet élevage (Gerber et al 2015) engendrent des critiques de la part des citoyens-consommateurs, critiques souvent virulentes, qui vont parfois jusqu'à remettre en cause l'existence de ces productions. Ces dernières concernent principalement les émissions de gaz à effet de serre, la faible efficacité des bovins à convertir les ressources naturelles en produits comestibles, la consommation et la pollution de l'eau ou encore l'utilisation des terres, et ceci en s'appuyant sur des chiffres qui tournent en boucle dans les médias et finissent par instiller le doute, comme, par exemple, « 15000 litres d'eau pour faire un kg viande » ou " $18 \%$ des émissions mondiales de gaz à effet de serre » ou encore « 15 kcal pour produire $1 \mathrm{kcal}$ de viande de ruminant $\gg$.

Dresser le bilan environnemental de cet élevage ne peut être réalisé sans considérer et prendre en compte la diversité des systèmes de production qui coexistent au sein du territoire français. Ainsi, les systèmes dits " naisseurs " sont de loin les plus nombreux, représentant près des $2 / 3$ des exploitations de bovins viande $(64 \%$ des exploitations ayant plus de 20 vaches allaitantes ; Institut de l'Élevage 2014). Ils produisent des mâles élevés sur des prairies permanentes vendus entre 5 et 9 mois (broutards), ou des mâles vendus plus tardivement (broutards repoussés) ou après une deuxième saison à l'herbe. Les systèmes dits «naisseursengraisseurs » représentent un petit tiers des exploitations (27\% des exploitations ayant plus de 20 vaches allaitantes). Ils englobent des exploitations qui engraissent leurs jeunes bovins (taurillons) à l'étable à partir de maïs ensilage et d'aliments concentrés $(17 \%)$, des exploitations $(8 \%)$ qui produisent des veaux élevés à partir du lait maternel (veaux sous la mère) ainsi qu'un très faible pourcentage d'exploitations qui produisent des bœufs engraissés au pâturage $(2 \%)$. Ces deux principaux systèmes produisent en outre une quantité importante de viande sous forme de vaches de réformes et de génisses de boucherie engraissées ou non. Enfin, un dernier système, peu présent sur le territoire français, est celui des « engraisseurs spécialisés » qui représentent seulement $1 \%$ des exploitations de bovins viande. Les agriculteurs achètent des broutards qu'ils engraissent à partir des cultures principalement produites sur leur exploitation.

L'ambition de cet article est de synthétiser les travaux scientifiques et les données statistiques disponibles sur les impacts environnementaux attribués aux exploitations françaises de bovins viande en les distinguant selon les types de système lorsque les données le permettent. L'entrée sera faite par type d'impact : les émissions de gaz à effet de serre, la pollution de l'eau et des sols, l'utilisation des ressources naturelles, l'utilisation des terres et la biodiversité végétale et animale. L'article se terminera par une analyse de l'évolution des performances environnementales des exploitations sur les vingt dernières années afin d'aborder le concept de durabilité des systèmes de bovins viande français.
1 / Les émissions de gaz à effet de serre et le stockage de carbone

L'ensemble des exploitations de bovins viande contribue aux émissions des trois principaux Gaz à Effet de Serre (GES) : le méthane $\left(\mathrm{CH}_{4}\right)$, le protoxyde d'azote $\left(\mathrm{N}_{2} \mathrm{O}\right)$ et le gaz carbonique $\left(\mathrm{CO}_{2}\right)$. Le méthane est essentiellement dû à la production de méthane entérique par les herbivores, presque exclusivement des ruminants, et secondairement à la fermentation anaérobie des effluents d'élevages de ruminants et de monogastriques. L'émission de $\mathrm{N}_{2} \mathrm{O}$ est principalement due à la production et à l'épandage d'engrais, et aux effluents d'élevage, fèces et surtout urine, sous forte influence du statut hydrique des sols récepteurs. Le gaz carbonique $\left(\mathrm{CO}_{2}\right)$ est quant à lui, produit sur la ferme (chauffage des bâtiments, carburants...) mais aussi en amont de la ferme (processus industriels et transports). Les cycles biologiques simplifiés du carbone et de l'azote sont résumés dans la figure 1.

\section{1 / Évaluation des émissions}

Mesurer directement toutes les émissions liées à une exploitation agricole ou à un système de production est impossible. Aussi, depuis une dizaine d'année, les émissions de GES par le secteur de l'élevage, qualifiées d'émissions brutes, sont évaluées et calculées par Analyse du Cycle de Vie (ACV) dont les principes et les méthodes de calcul sont spécifiés dans l'encadré. Les émissions sont alors rassemblées en un indicateur synthétique, l'équivalent $\mathrm{CO}_{2}$ (éq- $\mathrm{CO}_{2}$ ), qui correspond à un impact appelé « changement climatique " dans la terminologie des $A C V$, et qui tient compte du pouvoir 
Figure 1. Transferts de carbone (A) et d'azote (B) au pâturage entre la plante, l'animal, le sol et l'air : schémas simplifiés.
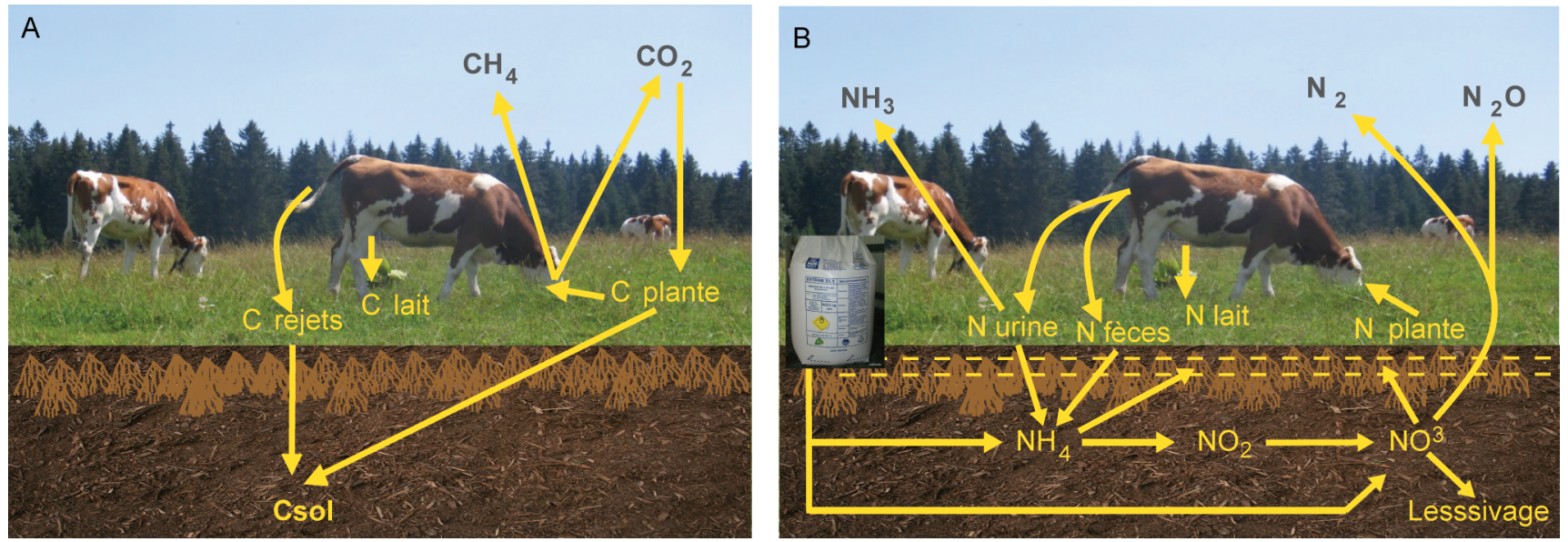

Encadré. L’analyse du cycle de vie (ACV), atouts et limites méthodologiques.

L'ACV prend en compte et quantifie toutes les sources d'émission dans les exploitations et en amont : production d'intrants (engrais, fourrages et aliments concentrés achetés...), transports à tous les stades de la production jusqu'à la ferme, changement d'usage des terres, en particulier déforestation inhérente à l'extension de la culture du soja. C'est un outil de réflexion plus que de classement de systèmes ou de produits. Le résultat est en outre fortement dépendant des hypothèses relatives aux facteurs d'émission. II permet une analyse en théorie multicritère, mais souvent réduit à l'examen d'un seul critère (généralement les émissions de GES). L'unité d'expression la plus répandue pour caractériser un impact environnemental est le $\mathrm{kg}$ de produit (lait ou viande), ce qui correspond à une vision globale, ayant comme finalité de nourrir l'humanité, ou, pour un pays, de maximiser sa production. Cependant de nombreux chercheurs utilisent également comme référence l'hectare, ce qui correspond à une vision territoriale : comment minimiser les impacts environnementaux potentiels par unité de surface au niveau d'une exploitation ou d'un territoire. Les conclusions sont différentes selon le mode d'expression (appelé aussi unité fonctionnelle dans la terminologie des ACV), mais ces deux visions sont complémentaires, même pour les impacts non localisés comme les GES.

Par ailleurs, à unité d'expression identique, les résultats relatifs à une même production sont parfois très différents d'une ACV à l'autre. Cela tient souvent aux hypothèses de départ (limites du système...), mais parfois aussi à des facteurs méthodologiques. L'un des principaux est l'allocation : Iorsqu'un produit a deux fonctions (huile et tourteau pour le soja) ou lorsqu'un système fournit deux produits (lait et viande pour le troupeau bovin laitier), comment allouer les impacts du système étudié entre ces produits? Dollé et al (2014) ont dénombré six modes d'allocation qui entraînent, pour la viande issue du troupeau laitier, des émissions brutes variant de 0 (allocation au produit majoritaire, très rarement pratiquée actuellement), à 4,4 (allocation à la production de protéines), 6,4 (allocation biologique, en fonction des besoins alimentaires des animaux), 7,5 (allocation selon la valorisation économique), 8,7 (allocation selon les phases de production, dans laquelle les phases de lactation et tarissement sont attribuées au lait), et $9,5 \mathrm{~kg}$ éq- $\mathrm{CO}_{2}$ par $\mathrm{kg}$ de produit (allocation par extension de système, qui attribue au $\mathrm{kg}$ de viande produit par le troupeau laitier l'émission du $\mathrm{kg}$ de viande produit par le troupeau allaitant). L'allocation économique répond à une logique, mais est souvent critiquée en raison de l'instabilité des marchés qui peut créer des biais lors de comparaison entre pays (Gac et al 2012, Salou et al 2017).

réchauffant relatif de ces trois gaz, par rapport à la référence que représente le $\mathrm{CO}_{2}$, à un horizon de 100 ans $\left(\mathrm{CO}_{2}=1\right.$, $\mathrm{CH}_{4}=25, \mathrm{~N}_{2} \mathrm{O}=298$ ).

Ces émissions brutes sont partiellement compensées par le stockage de carbone (C) dans le sol (émissions brutes moins stockage de $\mathrm{C}=$ émissions nettes). L'origine de ce stockage est la dégrada- manente a pendant longtemps été considéré comme compris entre 200 et $500 \mathrm{~kg}$ de $C$ par ha et par an avec des mesures d'évolution du C du sol dans les couches superficielles. Les nouvelles méthodologies utilisant des « tours à flux » qui incluent le stockage en profondeur font état d'une moyenne de $760 \mathrm{~kg}$ de C par ha et par an (Soussana et al 2014), avec une variabilité extrême, allant de plus de $2000 \mathrm{~kg}$ de stockage à un déstockage net dans certains cas. Les principaux facteurs de variations sont les caractéristiques pédologiques des sols, mais aussi les conditions climatiques et les pratiques agricoles (Klumpp et al 2010). Cette très forte variabilité, et donc l'impossibilité de quantifier avec précision le stockage de $\mathrm{C}$ pour un territoire ou pour un système d'élevage donné, fait qu'il n'est pris en compte ni dans les inventaires nationaux ni dans les études de la FAO, alors que son importance est désormais reconnue par le Giec, la FAO et par l'ensemble des acteurs scientifiques. Les premières études prenant en compte le stockage de $\mathrm{C}$ dans des ACV sont françaises et récentes (Doreau et al 2011, Veysset et al 2011), mais sont de plus en plus répandues et acceptées par la communauté scientifique. Elles intègrent des niveaux de stockage plus proches des nouvelles estimations, ainsi que le stockage par les haies, qui sont estimées à $125 \mathrm{~kg}$ de $\mathrm{C}$ par 100 m linéaires par an (Morel et al 2016).

\section{2 / Émissions en production de viande et facteurs de variation}

La FAO a évalué la contribution de l'ensemble des élevages au niveau mondial à 14,5\% des émissions anthropiques (Gerber et al 2013), et non plus à $18 \%$ comme cela avait été estimé auparavant (Steinfeld et al 2006). Ce chiffre est très supérieur à celui obtenu par une évaluation sectorielle comme celle qui est pratiquée dans les inventaires nationaux d'émissions. Le Citepa (2016) a ainsi estimé que le secteur de l'élevage contri- 
Tableau 1. Influence de la nature du régime distribué à des des taurillons à l'engraissement sur leurs impacts environnementaux (Doreau et al 2011 pour les gaz à effet de serre, Nguyen et al 2012a pour les autres impacts).

\begin{tabular}{|c|c|c|c|}
\hline Impact par kg de gain de poids & ${ }^{(1)}$ Régime $F$ & ${ }^{(1)}$ Régime EM & ${ }^{(1)}$ Régime C \\
\hline Gaz à effet de serre bruts ( $\mathrm{kg}$ éq- $\mathrm{CO}_{2}$ ) & 5,16 & 4,74 & 3,65 \\
\hline dont méthane entérique $\left(\mathrm{kg}\right.$ éq- $\left.\mathrm{CO}_{2}\right)$ & 3,33 & 3,81 & 1,56 \\
\hline Gaz à effet de serre nets ( $\mathrm{kg}$ éq- $\mathrm{CO}_{2}$ ) & $3,04-4,56$ & 4,74 & 3,65 \\
\hline Potentiel d'eutrophisation (g éq- $\mathrm{PO}_{4}$ ) & 16,5 & 19,0 & 21,5 \\
\hline Potentiel d'acidification (g éq-SO $\mathrm{S}_{2}$ ) & 31,3 & 31,8 & 29,4 \\
\hline Consommation d'énergie (MJ) & 18,7 & 13,0 & 19,7 \\
\hline Occupation de terres agricoles sur un an $\left(\mathrm{m}^{2}\right)$ & 11,7 & 4,5 & 4,6 \\
\hline dont terres arables $\left(\mathrm{m}^{2}\right)$ & 3,4 & 4,5 & 4,6 \\
\hline
\end{tabular}

${ }^{(1)}$ Régime $\mathrm{F}: 50 \%$ foin de prairie naturelle, $50 \%$ concentré ; régime $\mathrm{EM}: 65 \%$ ensilage de maïs, $35 \%$ concentré ; régime C : $86 \%$ concentré, $14 \%$ paille. Durée de l'engraissement : 6 mois.

buait à $9 \%$ des émissions en France. Calculés par ACV, les niveaux d'émissions à la sortie de la ferme sont de l'ordre de quatre fois plus élevées pour le $\mathrm{kg}$ de viande de bœuf que pour le $\mathrm{kg}$ de viande de porc et de poulet, ou de poisson selon l'étude française de référence AgriBalyse citée par Aubin (2014).

Concernant plus spécifiquement l'élevage de bovins viande, les travaux ont clairement montré que $90 \%$ des émissions brutes de GES par kg de carcasse de tous les animaux produits par l'ensemble du système (naisseur + engraisseur) est attribuable à la phase naisseur, ces émissions étant de l'ordre de $15 \mathrm{~kg}$ éq- $\mathrm{CO}_{2}$ par $\mathrm{kg}$ de poids vif produit (Nguyen et al 2012b). D'autres données font état d'une proportion un peu moins élevée de la phase naisseur. La part très importante du système naisseur est bien sûr due en grande partie au méthane entérique. Celui-ci est très globalement lié aux quantités d'aliments ingérés dans l'atelier naisseur par la vache allaitante et son veau, mais aussi par les génisses d'élevage. Berton et al (2016) montrent, en comparant les ateliers naisseurs en France et les ateliers d'engraissement en Italie, que les émissions restent plus élevées pour l'atelier naisseur que pour l'atelier engraisseur avec, respectivement, 15,1 et $9,6 \mathrm{~kg}$ éq- $\mathrm{CO}_{2}$ par kilo de poids vif produit. Les émissions de l'atelier naisseur étaient alors calculées par $\mathrm{kg}$ de broutard vendu pour l'atelier naisseur et par kg de gain de poids vif pour l'atelier engraisseur. Dans l'étude de Nguyen et al (2012b), les principaux postes d'émission brute de GES, après le méthane entérique $(40 \%)$, sont la production d'herbe, la gestion des effluents et la production d'aliments concentrés (respectivement 25,21 et $10 \%$ des émissions en moyenne). Les émissions liées aux bâtiments ou aux travaux sur la ferme ne totalisaient que $4 \%$.
Ces chiffres sont voisins de ceux retrouvés dans d'autres essais, avec toutefois des différences liées à la méthodologie utilisée : le méthane entérique représente 55 à $60 \%$ des émissions dans l'essai de Morel et al (2016) et de 58 à $67 \%$ dans l'essai de Veysset et al (2011). Les différences liées au système de conduite du troupeau allaitant sont extrêmement limitées : environ $4 \%$ entre des systèmes à vêlage d'automne ou de printemps (Morel et al 2016). Dans les systèmes engraisseurs, c'est le mode d'alimentation qui joue le rôle le plus important dans les émissions. Un exemple concret sur un système engraisseur de taurillons est présenté dans le tableau 1 : trois lots de taurillons ont été engraissés pendant 6 mois avec des rations à $65 \%$ d'ensilage de maïs et $35 \%$ de concentré (EM), classiques en France, $50 \%$ de foin de prairie permanente et $50 \%$ de concentré $(\mathrm{F})$, peu pratiquées jusqu'à présent, et $86 \%$ de concentré (essentiellement céréales) et $16 \%$ de paille (C), parfois utilisées en France mais surtout caractéristiques du système « feedlot » américain.

Ces émissions brutes sont largement à nuancer si l'on prend en compte le stockage de $\mathrm{C}$ dans les sols des exploitations. Les prairies représentent en effet une part importante de l'alimentation dans la plupart des exploitations et en particulier celles appartenant au système naisseur. Le calcul des émissions nettes est cependant très variable selon les hypothèses et les modes de calcul du stockage du $\mathrm{C}$ qui est, pour les raisons évoquées ci-dessus, plus faible pour les travaux les plus anciens. En proportion des émissions brutes, le stockage de $\mathrm{C}$ correspond à 13 à $21 \%$ en agriculture conventionnelle et 21 à $27 \%$ en agriculture biologique selon Veysset et al (2011). Un chiffre plus faible de 8 à $10 \%$ des émissions brutes a été obtenu par Nguyen et al (2012b) mais ces auteurs avaient intégré, outre le stockage de $\mathrm{C}$ par les sols, le relargage de $\mathrm{C}$ du sol lié à la déforestation de la forêt primaire pour produire du soja. Plus récemment, la compensation des émissions brutes par le stockage du C dans les sols a été estimée entre 24 et $53 \%$, selon les systèmes, par Dollé et al (2011), et entre 40 et $45 \%$ par Morel et al (2016). Il est donc clair que le stockage de $\mathrm{C}$ réduit fortement les émissions de GES, mais aussi qu'il est difficile de l'estimer avec précision.

\section{3 / Les pistes pour réduire les émissions de GES}

Récemment, une étude de l'INRA a analysé l'ensemble des pistes envisageables pour l'agriculture française sans changer de système de production. Plusieurs actions sont applicables au secteur de l'élevage de bovins viande : amélioration de la gestion des prairies (allongement, d'une part, de la durée annuelle de pâturage permettant entre autres de réduire la contribution des effluents d'élevage, et d'autre part, de la durée de vie des prairies temporaires), réduction de la fertilisation azotée, introduction de légumineuses (Klumpp et al 2013, Pellerin et al 2015). En revanche certaines actions préconisées dans cette étude sont peu utilisables pour les bovins viande. Ainsi, les voies efficaces permettant de faire baisser les émissions de méthane entérique comme l'apport de graines oléagineuses et de certains additifs (Doreau et al 2017) ne peuvent être utilisées que de manière limitée en production de viande, essentiellement pour les bovins en engraissement. De même la réduction du niveau des apports azotés dans la ration ou le semis de légumineuses sont des solutions plus praticables à grande échelle pour le troupeau laitier que pour le troupeau allaitant.

Dans le cas du troupeau allaitant, l'essentiel des impacts étant lié à la phase « naisseur », il est clair que la réduction 
Tableau 2. Impact potentiel de différentes pratiques d'élevage sur des critères environnementaux évalués par ACV et exprimés par kg de viande produite : essai de synthèse de la bibliographie(1).

\begin{tabular}{|c|c|c|c|c|c|}
\hline & $\begin{array}{c}\text { Emissions } \\
\text { de GES }\end{array}$ & $\begin{array}{l}\text { Stockage } \\
\text { de C }\end{array}$ & $\begin{array}{l}\text { Consommation } \\
\text { d'énergie }\end{array}$ & $\begin{array}{l}\text { Eutrophisation } \\
\text { Potentielle }\end{array}$ & $\begin{array}{l}\text { Acidification } \\
\text { Potentielle }\end{array}$ \\
\hline $\begin{array}{l}\text { Accroître le pâturage de prairies } \\
\text { permanentes }\end{array}$ & $=$ & התוג & & & \\
\hline $\begin{array}{l}\text { Diminuer le temps de présence en } \\
\text { bâtiments }\end{array}$ & ע & & & & \\
\hline Vêlage à 2 ans & ע & $=$ & y & $=$ & $=$ \\
\hline $\begin{array}{l}\text { Vêlage de printemps par rapport à } \\
\text { l'automne }\end{array}$ & & $\pi$ & ע & ע & ע \\
\hline $\begin{array}{l}\text { Finition de bœufs de } 2 \text { à } 3 \text { ans } \\
\text { par rapport à des taurillons }\end{array}$ & הת & $\pi$ & & $\pi$ & $\pi$ \\
\hline $\begin{array}{l}\text { Réduire la fertilisation azotée au } \\
\text { niveau des besoins }\end{array}$ & $=$ & $=$ & $=$ & ע & $=$ \\
\hline Agriculture biologique & C & הת & ע & C & C \\
\hline
\end{tabular}

(1) L'absence de flèche dans une case signifie l'absence d'étude spécifique. Le signe = indique soit que la pratique n'a pas d'effet ou a un effet mineur sur le critère considéré, soit qu'elle ne peut pas influencer ce critère. La lettre $C$ indique des effets opposés selon l'étude considérée. Ces effets opposés s'expliquent par des différences de méthodologie, ou de conditions de mises en œuvre de la pratique. Deux ou trois flèches dans une case correspondent à un effet marqué ou très marqué.

des impacts au niveau de l'ensemble du système passe par la réduction des impacts liés à la vache allaitante et à son veau, et aux génisses d'élevage. Comme l'atelier naisseur est essentiellement de type herbager, les pistes de réduction, plus que la réduction de l'émission du méthane entérique, sont liées à l'utilisation et à la gestion des ressources fourragères et à l'accroissement du stockage du C par le système. Un accroissement de la surface de prairies permanentes utilisée par Unité de Gros Bovin (UGB) entraîne une diminution linéaire des émissions nettes de gaz à effet de serre (Devun et al 2016). Des changements de système d'élevage peuvent aussi être envisagés, comme le choix du type d'animaux produits, taurillon ou bœuf : plus l'animal est élevé longtemps, plus il produit de méthane, et parfois des autres GES. De même le vêlage à 2 ans permet de réduire les émissions de GES du fait de la diminution de la phase d'élevage des génisses (Nguyen et al 2013a). L'ensemble des pistes envisageables en production de viande est détaillé dans le tableau 2.

Une réflexion similaire a été conduite dans d'autres pays. Ainsi Moran et al (2011) au Royaume-Uni et McKinsey \& Company (2009) aux Etats-Unis ont proposé des solutions allant dans le sens d'un accroissement de productivité, surtout en phase d'engraissement, et incluant des agents anti-méthanogènes inefficaces ou interdits dans l'Union Européenne. Les propositions irlandaises (Schulte et al 2011) sont plus proches dans leur esprit des propositions françaises (davantage de pâturage et moins d'azote minéral), mais incluent également des changements de système d'élevage : engraissement de taurillons à la place de bœufs pour diminuer la carrière des animaux et accroissement du troupeau laitier aux dépens du troupeau allaitant pour produire plus de viande à partir du troupeau laitier. Cette dernière mesure, dont il faudrait analyser toutes les conséquences sur la durabilité des filières d'élevage avant de la proposer, est celle qui serait la plus à même de réduire significativement les émissions de GES pour la production de viande. Son efficacité est liée à la constatation que la production de viande à partir du troupeau laitier en utilisant des races mixtes génère moins de GES par $\mathrm{kg}$ de viande (De Vries et al 2015), du fait de l'allocation des émissions en partie au lait. La fraction des émissions allouée à la production de viande est de l'ordre de 15 à $30 \%$ du total, selon le mode de calcul (Nguyen et al 2013b, Dollé et al 2014). Cependant, un redéploiement de l'élevage laitier avec ses caractéristiques actuelles au détriment de l'élevage de bovins viande pourrait conduire à une diminution des prairies permanentes, pourvoyeuses de biodiversité et de nombreux services écosystémiques, dont la régulation du climat via leur capacité à stocker du $\mathrm{C}$.

\section{2 / La pollution des eaux et des sols}

\section{1 / Les impacts potentiels sur l'eutrophisation et l'acidification}

Les sources de pollution de l'eau et des sols par les élevages sont très diverses : nitrate, nitrite, oxydes d'azote, phosphate, ammoniac et dioxyde de soufre. Comme pour les GES, les impacts de ces différents polluants peuvent être évalués par ACV. Ils sont alors regroupés en deux impacts environnementaux : l'acidification (des sols) et l'eutrophisation (des eaux). L'impact « acidification » regroupe les effets diffus des nitrites, oxydes d'azote, ammoniac et dioxyde de soufre considérés comme des acidifiants sur les sols, les eaux et les écosystèmes. Cet impact est pondéré dans une unité commune, le g d'équivalent-SO $\mathrm{S}_{2}$. L'impact " eutrophisation » est un critère du risque de prolifération d'algues vertes dans les eaux, intégrant les effets des nitrates, des nitrites, des oxydes d'azote, de l'ammoniac et des phosphates. Il est pondéré dans une unité commune, le g d'équivalent$\mathrm{PO}_{4}$. Un potentiel d'eutrophisation élevé ne signifie pas nécessairement qu'il y aura un impact réel sur les eaux, celui-ci dépendant non seulement de la charge polluante provenant des exploitations, mais aussi du taux de transfert de ces polluants dans les eaux et de la densité des élevages sur le territoire. Il faut noter 
que d'une manière générale, le potentiel d'acidification varie parallèlement au potentiel d'eutrophisation.

Globalement, les impacts des bovins viande quant aux potentiels d'eutrophisation et d'acidification sont importants. Ainsi Aubin (2014) évalue les potentiels moyens d'eutrophisation et d'acidification de la viande française de bœuf comme étant de l'ordre de 3 fois plus importants que ceux associés à la viande de porc et de poulet : respectivement 52,17 et $19 \mathrm{~g}$ équivalent $\mathrm{PO}_{4}$ et 147,40 et $45 \mathrm{~g}$ équivalent $\mathrm{SO}_{2}$. Cet impact élevé est confirmé sur l'acidification par l'étude de Martin et Mathias (2013). Ces auteurs évaluent à $40 \%$ la contribution des élevages des bovins viande aux émissions d'ammoniac liées à l'élevage qui représentent ellesmêmes $75 \%$ des émissions françaises d'ammoniac.

Sur le plan des systèmes d'élevage et si on considère ces impacts par kg de viande produit, la phase « naisseur » est responsable, comme pour les émissions de GES, de la part la plus importante des potentiels d'eutrophisation et d'acidification. Dans l'étude basée sur un système standard en zone charolaise avec engraissement des mâles, la part de la phase naisseur dans le potentiel d'eutrophisation était de près de $90 \%$, le principal poste d'émission étant la production d'herbe (60\% environ). Les deux autres postes significatifs étaient la production d'autres aliments et la gestion des effluents, à raison de $20 \%$ environ pour chacun d'eux (Nguyen et al 2012b). Cette part était de $80 \%$ dans le potentiel d'acidification avec $45 \%$ attribués à la production d'herbe et $35 \%$ à la gestion des effluents. Dans ces systèmes naisseurs, les vêlages d'automne entraînent des potentiels d'eutrophisation et d'acidification par $\mathrm{kg}$ de viande produit, pour l'ensemble du système, plus élevés que les vêlages de printemps, en raison d'intrants plus importants (Larue et al 2012, tableau 2). En revanche, le vêlage à 2 ans ne modifie pas les potentiels d'acidification et d'eutrophisation (Nguyen et al 2012b). Dans l'ACV restreinte à la période d'engraissement comparant 3 rations (tableau 1), le potentiel d'eutrophisation est plus élevé pour la ration très riche en concentré, toujours en raison des intrants plus élevés ; mais pour le potentiel d'acidification, le niveau est plus faible pour cette ration très concentrée, en raison d'émissions d'ammoniac par les effluents plus faibles. La production de bœufs pourrait entraîner des potentiels d'acidification et d'eutrophisation par $\mathrm{kg}$ de viande plus élevés que la production de taurillons, selon un unique essai mentionné par De Vries et al (2015); un âge d'abattage plus précoce des taurillons diminue également ces potentiels selon un essai portant sur un système japonais très spécifique (Ogino et al 2007). Il est donc possible de tirer quelques grandes tendances pour comparer des systèmes, mais la prédiction de différences entre systèmes d'alimentation reste difficile.

Lorsque les impacts sont exprimés par ha, les systèmes naisseurs basés sur l'herbe présentent d'une façon générale des potentiels d'eutrophisation et d'acidification les plus faibles, en particulier en raison d'un chargement moins élevé (Moreau et al 2013). Les systèmes en agriculture biologique présentent un potentiel d'acidification par kg de produit voisin ou plus élevé (De Vries et al 2015) que ceux des systèmes conventionnels lorsqu'ils sont exprimés par $\mathrm{kg}$ de produit, et inférieurs lorsqu'ils sont exprimés par ha. En revanche, deux études sur bovins viande relevées par De Vries et al (2015) font état de potentiels d'eutrophisation par $\mathrm{kg}$ de produit plus élevés en système biologique, probablement en raison d'une productivité animale inférieure et de la substitution de fertilisants minéraux par des fertilisants organiques. Ces résultats sont en désaccord avec ceux d'une revue de la littérature sur les bovins laitiers (De Boer 2003) qui avait montré pour les systèmes en agriculture biologique une potentiel d'eutrophisation tendant à être inférieur par $\mathrm{kg}$ de produit, et nettement inférieur par ha. Il est donc difficile de conclure, les résultats semblant très dépendants des conditions d'application de chacun des systèmes dans les études réalisées.

Une étude récente suggère des marges de progrès importantes de réduction des émissions d'ammoniac de l'élevage en France (Martin et Mathias 2013). Ces auteurs proposent deux leviers sur la conduite des animaux : le premier est l'optimisation de l'utilisation de l'azote alimentaire par l'animal afin de réduire les pertes, le second est l'allongement de la durée de pâturage, car la libération d'ammoniac dans l'air est plus faible au pâturage qu'en bâtiment, grâce à l'infiltration rapide de l'urine dans le sol et à la séparation physique entre urine et fèces. Ces mesures se traduisent en outre par un gain économique pour l'éleveur. Mais ils considèrent aussi que les techniques les plus efficaces concernent la gestion des effluents. Néanmoins, si la couverture des lisiers et des fumiers permet de réduire les émissions d'ammoniac, tout comme celles du $\mathrm{N}_{2} \mathrm{O}$, elle présente l'inconvénient d'accroître les émissions de méthane en raison des conditions anaérobies qu'elle promeut. Une solution à fort potentiel de réduction des émissions d'ammoniac serait l'enfouissement des effluents dans le sol, et non leur simple épandage.

\section{2 / Les pollutions liées aux pesticides}

Les polluants de synthèse tels que les pesticides (herbicides, fongicides et, à un degré moindre, insecticides) ont de nombreux effets, souvent mal connus, sur les écosystèmes et la santé humaine (Aubertot et al 2005). Malgré l'existence théorique d'un impact appelé « écotoxicité », ces molécules sont rarement prises en compte dans les ACV en raison de leur multiplicité et de la difficulté à hiérarchiser leurs effets. Kanyarushoki et al (2015) ont intégré cet impact dans l'ACV dans le cadre de l'élevage laitier et ont montré que le potentiel de toxicité terrestre par kg de lait, lié en particulier aux métaux lourds, était plus élevé dans des élevages plus intensifs à forte productivité (Finistère vs Cantal). Dans le cas de la production de viande, il est simplement possible de conclure à la faible utilisation de pesticides dans les exploitations en système naisseur ou en système naisseur-engraisseur de bœufs à l'herbe du fait de la proportion importante des prairies sur lesquelles l'utilisation des pesticides est très faible voire nulle. Au plan de la toxicité, ces exploitations ont probablement un impact limité sur les écosystèmes et produisent une viande contenant des résidus potentiels faibles. En revanche, la production de taurillons dans les systèmes naisseursengraisseurs et dans les systèmes engraisseurs faisant le plus souvent appel à une alimentation à base de cultures annuelles, il est probable, bien que non démontré, que l'éventuelle présence de résidus de pesticides dans la viande est liée à l'apport d'aliments autres que l'herbe : ensilage de maïs, céréales et tourteaux. Ainsi, selon l'étude britannique de Williams et al (2006), 97\% des pesticides utilisés pour la production de viande proviennent de l'épandage sur les matières premières constituant les concentrés.

\section{3 / L'utilisation de ressources naturelles}

Les activités agricoles sont consommatrices de diverses ressources naturelles, les plus souvent considérées étant l'énergie fossile et l'eau. À ces deux composantes, il faut ajouter le phosphore, car les engrais phosphatés constituent une des sources majeures de consommation de phosphore par les activités humaines.

\section{1 / L'énergie}

La consommation d'énergie a lieu dans l'élevage proprement dit (carburants des tracteurs, combustibles pour chauffer les bâtiments...) et en amont : énergie nécessaire à tous les processus industriels impliqués dans l'élevage (extraction des minerais, fabrication du matériel et des bâtiments agricoles, industrie des engrais et des aliments du bétail,...), et à tous les transports au long de la chaîne de production. Cette diversité de sources de 
consommation et la difficulté à les évaluer toutes pour caractériser un système d'élevage font qu'elle est également évaluée dans sa globalité grâce aux $\mathrm{ACV}$ qui se fondent sur une caractérisation de ces différents postes. Bien que ce soit l'énergie fossile qui soit la ressource à protéger, il est d'usage d'évaluer la consommation totale d'énergie, afin de faciliter les comparaisons entre essais et de mieux évaluer les postes les plus consommateurs. En effet, le pourcentage d'énergies renouvelables dans l'énergie produite varie d'un pays à l'autre et, pour un même pays, au cours du temps.

Comme pour les GES, la consommation d'énergie par $\mathrm{kg}$ de viande est plus élevée pour le bœuf que pour le poulet $(+20 \%)$ et surtout le porc avec 30,25 et $16 \mathrm{MJ}$ par kg, respectivement (Aubin 2014). Il y a déjà une décennie, il était montré que la consommation indirecte d'énergie, pour produire les engrais et aliments achetés en particulier, était supérieure à la consommation directe sur l'exploitation : fuel, électricité (Charroin et al 2006). Ces auteurs montraient aussi que les ateliers naisseurs étaient peu consommateurs d'énergie, par ha, comparés à des ateliers laitiers par exemple. En revanche, pour l'ensemble du système de production de viande, la part de l'énergie consommée par l'atelier naisseur par $\mathrm{kg}$ de produit est néanmoins comprise entre 73 et $83 \%$ selon les systèmes de production (Nguyen et al 2012b) et ce malgré la limitation des intrants dans un atelier naisseur ; l'énergie nécessaire à la production des fourrages et des concentrés est le poste le plus important.

Il y a de nombreux moyens de réduire la consommation d'énergie en production de viande sans changer de système d'élevage. Un premier levier est commun à toutes les exploitations et concerne l'utilisation du matériel : réglage des moteurs, adaptation de leur puissance, organisation des chantiers, choix des itinéraires culturaux (Couvreur 2006). Mais certains leviers sont spécifiques à la production de viande (tableau 2). Au niveau de l'ensemble du système, comme pour les émissions de GES et pour les mêmes raisons, l'avancement de l'âge au premier vêlage est une mesure relativement efficace : - 8\% selon Nguyen et al (2013a). Une différence beaucoup plus importante $(31 \%)$ a néanmoins été trouvée par Morel et al (2016) entre un système en vêlage de printemps économe et un système en vêlage d'automne qui l'est moins. Ce dernier entraîne une consommation accrue d'intrants achetés et la fourniture de fourrages conservés, alors qu'avec des vêlages de printemps la part d'herbe pâturée est plus élevée. La raison en est que le coût énergétique de la production d'herbe pâturée est plus faible que celui des fourrages conservés, lui-même plus faible que celui des céréales produites sur la ferme ; les concentrés achetés sont de loin les plus consommateurs d'énergie (Nguyen et al 2012a, tableau 1 et Nguyen et al 2012b). Les systèmes en agriculture biologique sont logiquement moins consommateurs d'énergie par $\mathrm{kg}$ de viande produite; la différence ne porte pas sur la consommation directe des exploitations (fuel, électricité), mais bien sur la diminution du poste « engrais de synthèse ». Quant à l'énergie nécessaire pour produire les aliments achetés, elle ne diminue pas nécessairement dans un système en agriculture biologique ; cela dépend des pratiques d'alimentation et d'engraissement. Enfin, comme pour les GES, la consommation d'énergie par $\mathrm{kg}$ de viande serait plus faible lorsqu'elle est produite à partir du troupeau laitier qu'à partir d'un troupeau allaitant spécialisé, car les consommations sont réparties entre les produits lait et viande.

\section{2 / L'eau}

La consommation d'eau par les activités agricoles représenterait $70 \%$ de l'eau utilisée pour les activités humaines, selon la FAO (Steinfeld et al 2006). Le réchauffement climatique et l'accroissement des sécheresses estivales risquent d'augmenter le recours à l'irrigation et le problème de concurrence pour l'accès à l'eau. L'élevage est ainsi mis en cause pour sa contribution à l'utilisation des ressources en eau, en particulier la production de viande. Le chiffre choc de 15000 L d'eau nécessaires pour produire un $\mathrm{kg}$ de viande est régulièrement repris dans de nombreux médias. L'évaluation de la méthodologie utilisée a été faite par Corson et Doreau (2013) et actualisée récemment avec un focus sur la production de viande (Doreau et Corson 2017). En fait, le calcul de l' « empreinte eau » qui aboutit à ce chiffre est à distinguer de la consommation réelle d'eau par les animaux et plus globalement par les élevages. La méthodologie de calcul de l'empreinte eau a été détaillée par Hoekstra et al (2011). Cette empreinte eau est la somme de l'eau bleue (eau réellement consommée, par exemple abreuvement, irrigation, nettoyage...), de l'eau verte (vapeur d'eau correspondant à l'évapotranspiration des surfaces agricoles destinées à la production de viande, celle-ci étant liée à la production de biomasse et d'autant plus élevée que la pluviométrie est importante) et de l'eau grise (eau virtuelle qui serait nécessaire pour dépolluer l'eau polluée par l'élevage). L'eau verte représente plus de $90 \%$ de l'empreinte eau, le reste se partageant entre l'eau bleue et l'eau grise. La figure 2 résume les valeurs calculées par Mekonnen et Hoekstra (2010) pour différents pays et systèmes d'élevage, et permet de comprendre de quoi se composent les 15000 L d'eau annoncés. Une très grande variabilité est observée en fonction des pays et des systèmes d'élevage. De même, certains chiffres sont étonnants : eau bleue et eau grise à zéro alors que l'eau verte est extrêmement élevée dans un système indien. Il est regrettable qu'aucun détail ne soit donné sur les systèmes étudiés.

Le calcul de l'eau grise a, jusqu'à présent, été réalisé uniquement en considérant la pollution potentielle par les nitrates, mais pas par les autres polluants (phosphates, composés organiques de synthèse). De par son mode de calcul, il ne présente pas la même rigueur que le calcul de l'eutrophisation par ACV, et si son utilisation au niveau des rejets d'une usine est facile à mettre en œuvre, elle l'est difficilement au niveau des processus globaux d'élevage car la pollution des nappes, eaux dormantes et rivières, par une exploitation agricole est diffuse.

Figure 2. Consommation d'eau selon la méthode de l'empreinte eau : proportions moyennes d'eau bleue, verte et grise pour 7 pays et 3 systèmes d'élevage (d'après Mekonnen et Hoekstra 2010).

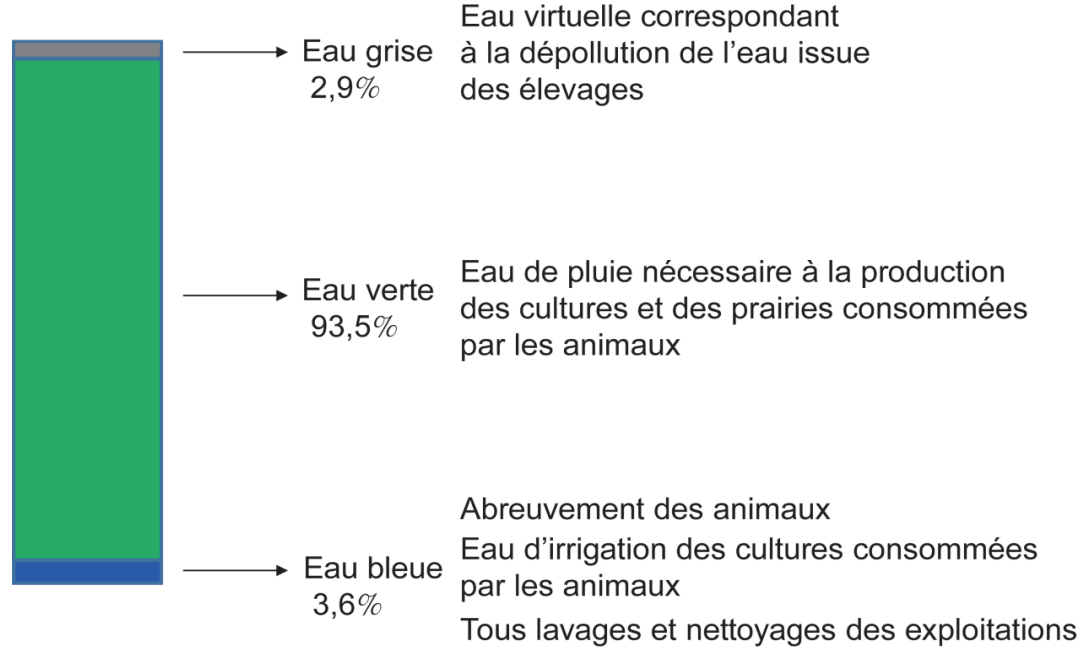

Eau virtuelle correspondant

à la dépollution de l'eau issue des cultures et des prairies consommées par les animaux 
Contrairement à l'eau grise, l'eau verte participe au cycle de l'eau au niveau de la planète et ne correspond donc pas à une consommation nette d'eau par les animaux. Son évaluation est utile pour raisonner l'utilisation des surfaces agricoles dans une zone géographique, et les échanges éventuels de produits agricoles entre zones arides et zones arrosées. Mais la prise en compte de l'eau verte dans le calcul de l'eau nécessaire pour produire $1 \mathrm{~kg}$ de viande est spécieuse : ainsi plus un système est extensif, plus il entraîne une " empreinte eau » élevée : le chiffre de $200000 \mathrm{~L}$ par kg de viande a même été avancé pour une production sur des surfaces sous-exploitées, utilisées comme parcours, pour lesquelles le besoin en eau verte par ha serait identique si ces surfaces restaient inutilisées.

On comprend donc que seule l'eau bleue réellement consommée dans les élevages peut être reliée à la pénurie d'eau. Les consommations sont très variables d'un système à l'autre, et d'un pays à l'autre ; mesurées par la méthode de l'empreinte eau (fraction eau bleue seule), elle serait voisine de $550 \mathrm{~L} / \mathrm{kg}$ de viande bovine, et du même ordre de grandeur que les consommations par $\mathrm{kg}$ de viande de porc ou de volaille (Mekonnen et Hoekstra 2010). En France, selon les premières estimations de Gac et Béchu (2014) par ACV, elles seraient de l'ordre de 240 à $330 \mathrm{~L} / \mathrm{kg}$ de viande bovine selon les systèmes d'élevage. Ces calculs concernent la consommation effective d'eau, mais ne permettent pas de juger du risque de pénurie en eau. Afin de mieux prévoir ce risque, une équipe australienne a conçu un calcul de l'eau bleue utilisée en tenant compte de l'eau consommée qui retourne au bassin versant, par exemple l'eau urinée, ou l'eau de lavage des bâtiments, et en établissant un système de pondération de la quantité d'eau bleue en fonction de l'état des réserves en eau dans la région, plus précisément au niveau du bassin versant (Ridoutt et Pfister 2013). Ce type de calcul conduit, dans une comparaison de systèmes de production de viande dans 6 zones géographiques d'Australie, à une quantité d'eau bleue utilisée variant de 25 à $234 \mathrm{~L} / \mathrm{kg}$ de viande et à une quantité d'eau bleue pondérée variant de 3 à $221 \mathrm{~L} / \mathrm{kg}$ de viande (Ridoutt et al 2012). Il est clair qu'une réflexion sur la signification des chiffres avancés est indispensable, plus encore dans le domaine de la consommation d'eau que pour d'autres impacts environnementaux.

\section{3 / Le phosphore}

L'utilisation de phosphates dans l'agriculture a non seulement un effet sur la qualité des eaux, mais aussi sur la raréfaction de ressources naturelles. On considère que les ressources naturelles de phosphates connues à l'heure actuelle et accessibles à un prix raisonnable permettent de maintenir la consommation actuelle pendant une centaine d'années (Castillon 2005). Toutefois, la raréfaction de la ressource nécessite de connaître son utilisation en élevage, incluant l'utilisation pour la production d'aliments concentrés achetés, et les moyens de la réduire. Selon des données de la base Agreste, la consommation d'engrais sous forme d'acide phosphorique en France diminue depuis l'année 2000 : elle est passée de 1 million à $600000 \mathrm{~T}$ d'acide phosphorique entre 1999 et 2006 . Il n'y a pratiquement pas de références concernant l'utilisation de phosphore spécifiquement pour la production de viande bovine. Une étude australienne mentionne qu'un $\mathrm{kg}$ de phosphore permet de produire 16,31 et $52 \mathrm{~kg}$ de viande de bœuf, de porc et de volailles, $232 \mathrm{~kg}$ de lait, $344 \mathrm{~kg}$ de céréales et $2500 \mathrm{~kg}$ de protéagineux (Metson et al 2016). Bien qu'il n'y ait pas d'étude spécifique en France et que la répartition des ventes d'engrais phosphatés entre filières ne soit pas disponible, il est probable qu'en France la viande de bœuf nécessite des quantités de phosphore beaucoup plus faibles, car le mode d'exploitation du troupeau allaitant ne requiert pas une fertilisation phosphatée élevée, la fertilisation moyenne d'un ha de prairie permanente étant inférieure à celle d'un ha de blé.

\section{4 / L'utilisation des terres}

Le sol est une ressource d'autant plus précieuse que, face à la demande croissante de nourriture pour l'humanité, en particulier de produits animaux, le besoin théorique en terres agricoles s'accroît. Certes il y a, en particulier en Afrique, un potentiel de terres actuellement sousexploitées, mais leur mise en culture ou leur utilisation plus intensive pose des problèmes liés à la maîtrise du foncier. Parallèlement, l'utilisation des terres est menacée par l'urbanisation croissante, qui semble irréversible, et par le développement des agrocarburants. Or la production d'un kg de protéines animales, en particulier sous forme de viande, nécessite beaucoup plus de surface qu'un $\mathrm{kg}$ de protéines végétales. Parmi les viandes, $1 \mathrm{~kg}$ de viande bovine nécessite 5 fois plus de surface qu' $1 \mathrm{~kg}$ de viande de porc ou de volaille (Aubin 2014). La forte demande en terres agricoles est, comme pour les autres impacts analysés, essentiellement due à la phase d'élevage allaitant $(95 \%$ en moyenne selon Nguyen et al 2012b). Elle est plus élevée avec des systèmes extensifs et des systèmes en agriculture biologique, qui se caractérisent par une part plus importante de l'herbe dans l'alimentation, et/ou par une productivité plus faible (De Vries et al 2015).
L'une des caractéristiques de la production de viande bovine est cependant qu'une partie des sols nécessaires est constituée de terres non arables, qui ne sont pas en compétition avec l'alimentation humaine ou l'alimentation des monogastriques. C'est le cas des zones de montagne, et des zones où la qualité des sols ou le climat ne permettent pas de produire des céréales avec des rendements permettant d'assurer la rentabilité de la culture; de telles zones strictement herbagères sont nombreuses en France. La production de viande sur ces surfaces est souvent une alternative à la forêt tout en contribuant à l'alimentation humaine (De Vries et al 2015). Lorsque ces herbages sont constitués de prairies permanentes, ils stockent du $\mathrm{C}$, et jouent donc un rôle positif dans la régulation des impacts environnementaux. Il est donc clair qu'il ne faut pas considérer dans l'évaluation d'un système d'alimentation toutes les surfaces de la même manière, et qu'une prairie permanente de montagne n'a pas le même rôle, en termes d'utilisation des surfaces agricoles, qu'une prairie temporaire de plaine. Toutefois même si des élevages de zone de plaine utilisent des herbages, dans le cas de zone de pratique de la polyculture-élevage, leur contribution à la fumure organique des terres arables constitue un service environnemental qui doit être mis en regard de l'impact négatif sur l'utilisation des terres (Dumont et al 2016).

La réflexion sur l'utilisation des sols au niveau national et au niveau mondial doit intégrer le découplage entre lieu de production des animaux et lieu de production des aliments qu'ils consomment. Un raisonnement local en termes de surface agricole utilisée par l'élevage est insuffisant. Le cas le plus emblématique est celui de l'engraissement en Italie de broutards produits en France : la surface nécessaire pour produire un broutard de $350 \mathrm{~kg}$ est bien supérieure à la surface nécessaire pour conduire ce broutard de 350 à $700 \mathrm{~kg}$, car la première phase nécessite des surfaces herbagères importantes, notamment pour alimenter la mère. La surface nécessaire pour produire $1 \mathrm{~kg}$ de viande doit dès lors prendre en compte l'ensemble du cycle, à l'instar des autres impacts environnementaux.

\section{5 / La biodiversité}

\section{1 / Les prairies permanentes, élé- ment structurant des exploitations de bovins viande et du paysage}

L'élevage des herbivores a contribué depuis plusieurs millénaires à créer une grande variété d'habitats semi-naturels, souvent caractérisés par des niveaux riches en biodiversité (Orlandi et al 2016). 
Les prairies permanentes figurent en particulier parmi les habitats les plus riches en biodiversité en Europe (Norderhaug et al 2000, Dengler et al 2014). Leur seule présence dans les exploitations peut à ce titre être considérée comme un indicateur de biodiversité.

Ces prairies couvrent néanmoins une gamme très large de situations, depuis des prairies fortement fertilisées et intensément exploitées jusqu'à des parcours à faible chargement animal par hectare. Ces deux facteurs, fertilisation et intensité d'utilisation du couvert végétal, permettent d'expliquer leur niveau de diversité floristique et faunistique (Cruz et al 2002). L'exploitation intense d'une prairie (par exemple fertilisation élevée combinée à un nombre de fauches ou à un chargement important) favorise des espèces végétales très compétitives à forte capacité de capture des éléments nutritifs et à vitesse de croissance et de renouvellement des feuilles élevée, espèces qui éliminent la plupart des autres et conduisent à un appauvrissement de la diversité végétale (Farruggia et al 2008). En revanche, une exploitation très modérée, combinant, par exemple, une fertilisation et un chargement faible ou une fauche tardive, favorise les espèces peu compétitives qui présentent des taux de croissance faibles et permet une cohabitation d'un nombre élevé d'espèces végétales. Du point de vue de la structure du couvert prairial pâturé, lorsque le niveau de chargement animal est faible à modéré, la valorisation de l'herbe par les animaux est partielle. Dans cette situation, les animaux préfèrent réutiliser, au sein du couvert, les zones qu'ils ont préalablement défoliées qui présentent les meilleures valeurs nutritives et délaissent les autres zones épiées, non pâturées en début de saison (Adler et al 2001). Cette hétérogénéité structurale permet la coexistence d'un grand nombre d'espèces de plantes associé à grande diversité faunistique. Dans l'herbe haute, les insectes bénéficient en effet d'un microclimat et de ressources alimentaires abondantes, ils sont mieux protégés des prédateurs et subissent moins les effets directs du pâturage (Pöyry et al 2006, Wallis de Vries et al 2007, Dumont et al 2009). Certaines espèces d'oiseaux y trouvent des habitats protégés pour la nidification, mais aussi des ressources alimentaires abondantes liées à l'augmentation des arthropodes (Benton et al 2002). A contrario, l'exploitation des prairies à des niveaux élevés de chargement associés à des stratégies de gestion optimisées de la pousse de l'herbe conduisent à la sélection d'un petit nombre d'espèces végétales compétitrices ainsi qu'à une homogénéisation du couvert avec une végétation rase peu favorable à une diversité d'espèces animales. Le suivi de prairies de moyenne montagne

Figure 3. Pourcentage de STH dans la SAU (A) et chargement animal (UGB/ha $S F P)^{(1)}(B)$ dans les exploitations "Bovins viande " ou "Bovins lait »(2) en France et dans les régions caractérisées par une présence importante des bovins viande (Source : Agreste, 2015 : données du Réseau d'Information Comptable Agricole (RICA), Année 2014 pour l'ensemble des moyennes et grandes exploitations).

(1)STH : Surface Toujours en Herbe ; SAU : Surface Agricole Utile ; UGB : Unité de Gros Bovins ; (2)Les classifications " bovins viande » et « bovins lait » se réfèrent à une typologie des exploitations agricoles propre au Réseau d'Information Comptable Agricole (RICA) fondée sur l'orientation technico-économique des exploitations (Otex) définie à partir de leur production principale. Bovins lait et bovins viande sont les appellations correspondant respectivement aux Otex 45 et 46.
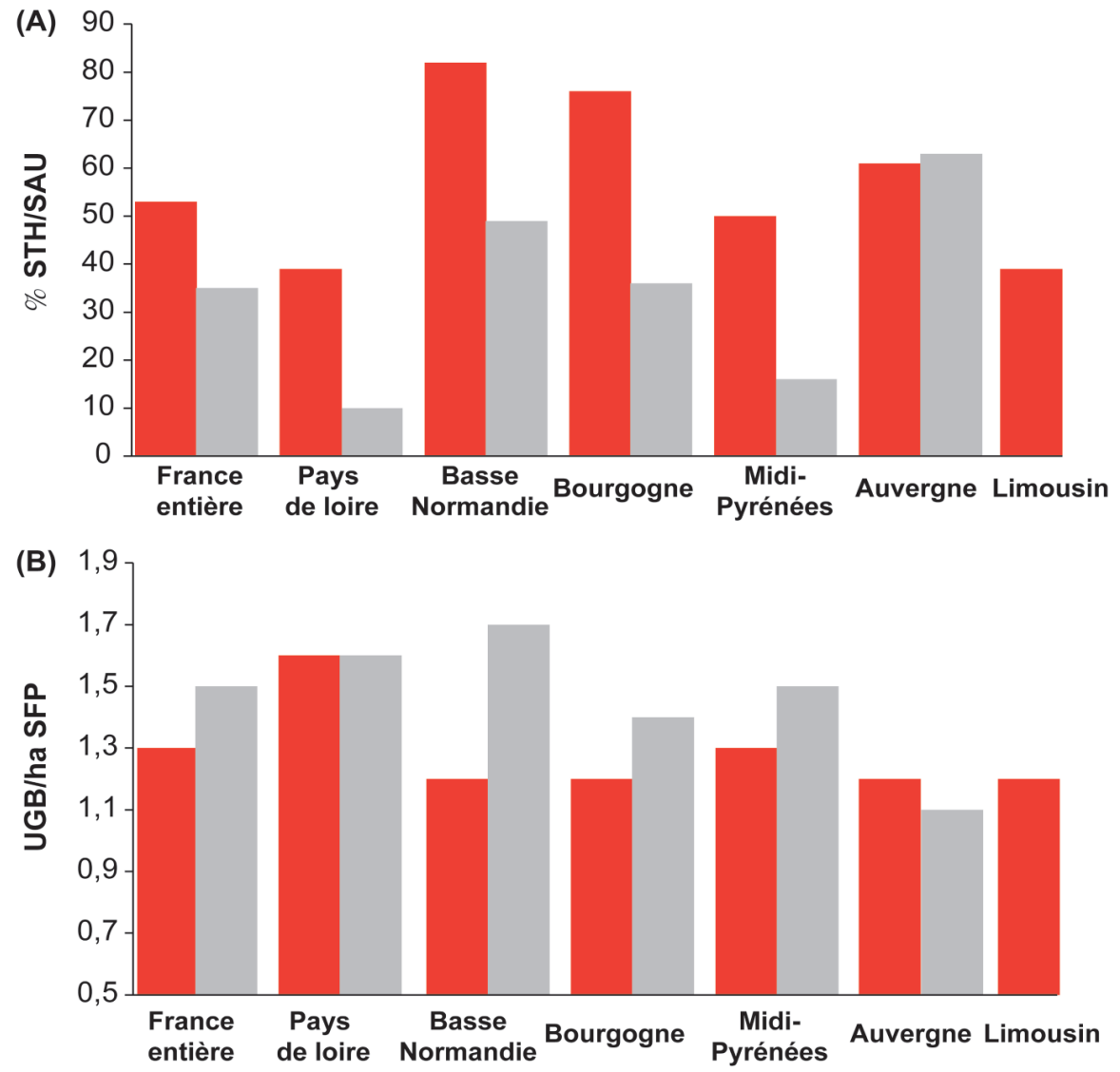

Bovins viande Bovins lait

exploitées par des génisses allaitantes dans un gradient de chargement $(0,5$; 0,85 et $1,2 \mathrm{UGB} / \mathrm{ha}$ ) a permis de montrer que l'abondance des plantes à fleurs avait significativement augmenté en 6 ans sur les parcelles à faible chargement tandis que les populations d'insectes avaient réagi très favorablement aux différences de structures de végétation directement liées au chargement (Dumont et al 2009). Le chargement global à l'échelle de l'exploitation peut également être un indicateur de la biodiversité présente sur l'exploitation. Dans des exploitations en système herbager allaitant du nord Cantal, situées à même altitude dans un même environnement pédoclimatique et paysager, le nombre d'espèces prairiales diminue avec l'augmentation du chargement des exploitations. Dans cette étude, il a également été montré que dans les exploitations les plus intensives, les pratiques mises en œuvre par les éleveurs visent à homogénéiser les parcelles et à réduire ainsi leur variabilité spatiale pour augmenter la productivité par hectare, réduisant de ce fait le nombre de faciès de végétation intra parcellaire et in fine la diversité floristique de la parcelle (Farruggia et al 2006).

Concernant plus spécifiquement les élevages de bovins viande, ils se distinguent par la part encore importante des surfaces toujours en herbe. Cette importance des prairies s'explique par la très forte dominance des systèmes naisseurs qui fondent l'élevage des jeunes animaux sur l'herbe. En France métropolitaine, $1 / 5$ des exploitations ayant plus de 20 vaches allaitantes sont exclusivement herbagères (Institut de l'Élevage 2014). Plus globalement, si on sépare les exploitations bovines selon la classification du Réseau d'Information Comptable Agricole (RICA) en fonction de leur orientation technico-économique (OTEX), la surface toujours en herbe 
(STH) représente en moyenne plus de la moitié de la surface agricole utile des exploitations classées « bovins viande » contre un tiers seulement dans les exploitations classées «bovins lait ». Cette part moyenne varie de $39 \%$ en Pays de Loire à $82 \%$ en Basse Normandie (figure 3a).

Le niveau d'intensification des surfaces fourragères, exprimé en UGB/ha SFP, peut également être considéré comme un indicateur frustre du niveau de diversité floristique et faunistique. Ainsi, le niveau de chargement moyen à l'échelle de la France dans les exploitations ayant des bovins viande peut être considéré comme relativement modéré avec 1,3 UGB/ha SFP. Il est inférieur à celui des exploitations ayant des bovins lait (1,5 UGB/ha SFP) sauf dans les pays de Loire, où il s'élève à $1,6 \mathrm{UGB} /$ ha et traduit le niveau d'intensification plus fort des exploitations naisseurs-engraisseurs de l'Ouest de la France (figure 3b). Dans toutes les autres régions dans lesquelles la concentration en vaches allaitantes est importante, le chargement moyen est compris entre 1,2 et $1,3 \mathrm{UGB} /$ ha $\mathrm{SFP}$ (Réseau d'Information Comptable Agricole 2014). L'élevage de bovins viande serait donc, en moyenne, plus favorable à la biodiversité que l'élevage laitier à l'exception des systèmes naisseursengraisseurs qui présentent de forts niveaux de chargements, alliés à la présence de maïs, et des systèmes naisseurs de montagne qui ont des chargements équivalents à ceux des systèmes laitiers.

Enfin, il est reconnu que l'élevage des herbivores façonne fortement les paysages dans lesquels il est implanté et contribue à créer des paysages ouverts et attractifs. La persistance de ces paysages diversifiés et hétérogènes, incluant des prairies permanentes, est considérée par beaucoup d'auteurs comme un élément clé pour maintenir la biodiversité dans les systèmes agricoles tempérés (MacDonald et al 2000, Benton et al 2003, Pykälä 2003). Luoto et al (2003) mettent clairement en évidence un changement important de la structure du paysage d'une vallée en Finlande après la forte diminution du pâturage sur des prairies permanentes à niveaux modéré de chargement au cours d'une période de 30 ans. Les pâtures, auparavant bien connectées entre elles, créant un long corridor et participant largement à l'hétérogénéité globale du paysage, ont laissé la place à des ilots isolés de prairies permanentes. Ce changement rapide de paysage a conduit à une diminution de la diversité floristique. Ces résultats illustrent l'importance des systèmes bovins viande qui au travers du pâturage des prairies permanentes permettent de maintenir une mosaïque d'habitats hétérogènes et une diversité de couverts végétaux à l'échelle des territoires.

\section{2 / Aptitude de l'espèce et diver- sité des races allaitantes}

Au niveau de l'animal, deux facteurs peuvent être possiblement pris en considération pour discuter de l'impact de l'élevage de bovins viande sur la biodiversité : le type d'espèce et le nombre de races présentes au sein de l'espèce considérée. Ainsi les bovins présentent des atouts vis-à-vis de la biodiversité des prairies. Leur faible capacité de tri dans les couverts pâturés due à la forme de leurs mâchoires, associée à leur grand volume de fermentation dans le rumen, leur permet de digérer des couverts fibreux et les rend moins sélectifs que les petits ruminants (Dumont et al 2007). Le pâturage bovin engendre également plus d'hétérogénéité de structure sur le couvert prairial que celui des ovins (Vickery et al 2001, Dumont et al 2011), ce qui peut conduire à une plus grande diversité floristique et faunistique.

Pour ce qui concerne les races, même si trois races dominent très largement le troupeau allaitant français (la Charolaise, la Limousine et la Blonde d'Aquitaine), il existe une variabilité génétique intrarace beaucoup plus importante que dans le troupeau laitier en raison du nombre important de taureaux pères. Par ailleurs, il y a encore un nombre important de races bovines, mixte ou viande. La Direction Générale des Politiques Agricoles, Agroalimentaire et des Territoires a ainsi recensé 61 races éligibles pour la prime aux bovins allaitants en France pour 2015. Ce chiffre intègre néanmoins une grande diversité de situations, notamment pour les races locales menacées d'abandon. Certaines, en particulier dans le Sud-Ouest et les Pyrénées comptent moins de 300 femelles comme par exemple la Bordelaise, la Béarnaise ou la Lourdaise (http://www.agroparistech.fr/svs/genere/especes/bovins.htm). A l'opposé, la race auvergnate Ferrandaise, en voie de disparition il y a 40 ans compte désormais plus de 1500 femelles réparties dans plus de 200 élevages grâce à une association de sauvegarde. La race Rouge des prés quant à elle s'est confortée en s'adossant à une AOP. Dans les prochaines décennies, la vision nouvelle de l'élevage par le consommateurcitoyen devrait contribuer au maintien des races locales variées même en l'absence de signe officiel de qualité.

\section{3 / Les pistes d'amélioration de la biodiversité}

Trois stratégies adaptées à trois profils différents d'éleveurs pourraient être mises en œuvre pour augmenter la biodiversité dans les exploitations.

La première stratégie, adaptée au plus grand nombre d'éleveurs, consisterait à proposer aux éleveurs des pratiques ayant peu d'impact sur la production ou empiétant peu sur l'espace productif. Dans la plupart des cas, il s'agirait d'implanter ou de préserver des zones dans lesquelles les plantes à fleurs (bandes fleuries en bordure de parcelles) peuvent se reproduire et offrir des abris et des ressources à la micro et à la macro faune (Luka et al 2016, Tschumi et al 2016). En prairie pâturée, Farruggia et al (2012) ont montré l'intérêt de la « rotation écologique» sur les populations de papillons sans que cela n'affecte les performances des génisses allaitantes. Cette conduite consiste à soustraire de la rotation une des parcelles au moment de la floraison de la majorité des dicotylédones, de mai à début août, afin de permettre aux plantes à fleurs de mieux réaliser leur cycle biologique et d'offrir aux insectes des abris et un habitat de qualité ainsi qu'une nourriture abondante.

Une deuxième stratégie préconiserait une meilleure intégration de la biodiversité dans les processus de production agricole avec une vision de la biodiversité en tant que ressource substituable aux intrants. Les travaux et outils issus des RMT (Réseau mixte technologique) et des projets Casdar (Compte d'affectation spéciale « développement agricole et rural ») centrés sur les connaissances multicritères incluant la biodiversité des prairies permanentes et temporaires pourraient être valorisés dans ce cadre (RMT « prairies demain », Casdar Prairies AOP, Casdar Prairies permanentes...). Toutefois, il serait pertinent que la Recherche et les acteurs accompagnant le monde agricole se mobilisent davantage sur l'agroécologie pour montrer aux éleveurs le bénéfice économique potentiel de promouvoir la biodiversité dans leur ferme.

Enfin, la troisième, non exclusive avec la précédente, viserait un éleveur déjà sensibilisé à la nature et ferait en sorte que l'éleveur acquière une meilleure connaissance des espèces végétales et animales présentes sur ses parcelles. Un travail d'inventaire botanique et faunistique avec l'aide d'organismes ou d'associations locales lui permettrait de mieux comprendre l'effet de ses pratiques sur les espèces et de mieux percevoir les enjeux de son territoire afin de devenir « conservateur».

\section{6 / Évolution des performances environnementales et de la durabilité des systèmes bovin viande}

Les évolutions techniques et économiques des exploitations allaitantes ont été analysées sur une durée de 24 ans 
(1990-2013) en s'appuyant sur deux réseaux : les exploitations « bovins viande » du RICA et 43 exploitations allaitantes du bassin Charolais de l'observatoire Inra suivies depuis 1990 (Veysset et al 2015). Les évolutions structurelles les plus importantes observées entre 1990 et 2013 sont une augmentation concomitante de la taille des exploitations et des troupeaux, à main-d'œuvre constante, entrainant une très forte augmentation de la productivité du travail. Sur le réseau national, un travailleur agricole gère aujourd'hui 75 ha de SAU et 80 UGB alors qu'il gérait 42 ha et 45 UGB en 1990. Le chargement animal s'est maintenu à $1,23 \mathrm{UGB} / \mathrm{ha} \mathrm{SFP}$. Ces augmentations de SAU et de productivité par travailleur sont les plus importantes d'Europe (cf. Lherm et al 2017 ce numéro).

Dans le réseau du Charolais, sur le plan de la conduite des surfaces fourragères, c'est l'augmentation de la part de surface d'herbe fauchée qui constitue l'évolution la plus importante (38 à $47 \%$ de 1990 à 2013). Elle est associée à une augmentation de l'utilisation des balles rondes enrubannées au détriment du foin (parts de la surface en herbe fauchée enrubannée : $7 \%$ en $1990,21 \%$ en 2013). Cette augmentation des surfaces en fauche, théoriquement précoce, conduit à une perte de biodiversité dans les exploitations du fait de la diminution des surfaces naturellement hétérogènes (pâtures) et de la perte de surfaces riches en épis (surfaces récoltées en foin) qui offraient plus de ressources alimentaires pour la faune et permettait à une large gamme d'espèces prairiales de se reproduire. En revanche, une très légère diminution de la consommation des engrais azotés (50 à $43 \mathrm{~kg} / \mathrm{ha}$ ) a été constatée. Sur le plan de la conduite des animaux, malgré la probable meilleure valeur alimentaire des fourrages (enrubannage), c'est l'augmentation des quantités de concentrés distribués par $\mathrm{kg}$ de poids vif qui constitue l'évolution la plus marquante : de 1,63 à $2,18 \mathrm{~kg}$ de concentré par $\mathrm{kg}$ de poids vif, soit $+33 \%$ en 24 ans ; les concentrés achetés et les concentrés produits sur la ferme pour produire $1 \mathrm{~kg}$ de poids vif ayant augmenté dans les mêmes proportions. Ces évolutions se sont accompagnées d'une diminution de la productivité numérique (nombre de veaux nés pour 100 vaches mises à la reproduction), d'une augmentation de la mortalité des veaux $(7,5$ à $9,5 \%)$ et d'une légère augmentation du $\mathrm{kg}$ de poids vif par UGB $(+6 \%)$ due à la sélection génétique et à l'apport plus important de concentré.

Sur le plan économique, l'efficacité technico-économique des systèmes a diminué : les volumes de viande et de cultures produits par ha de SAU sont restés stables alors que toutes les consommations intermédiaires par hectare ont augmenté à l'exception des engrais chimiques (concentrés, frais vétérinaires, carburant, réparation et entretien de l'équipement) et que le capital fixe annuel (hors capital cheptel) par ha SAU a également augmenté, les dépenses d'équipement étant la principale cause de cette augmentation en représentant plus de $60 \%$ du capital fixe par ha SAU. Cette diminution d'efficience des systèmes est à l'origine d'une hausse de la consommation d'énergie fossile par $\mathrm{kg}$ de poids vif produit de $15 \%$ (plus d'intrants et de capital pour le même volume de production). Les émissions de GES par $\mathrm{kg}$ de poids vif produits ont, quant à elles, légèrement baissé en raison de la légère augmentation de la productivité animale constatée, et donc de la dilution du méthane émis.

Enfin, Veysset et al (2016) ont par ailleurs, montré que parmi la diversité des élevages de bovins viande, les systèmes herbagers et/ou à faibles intrants montrent d'encourageantes, voire meilleures, performances comparativement aux systèmes polyculture-élevage de plaine et ce sur les trois dimensions du développement durable.

\section{Conclusion}

Cette synthèse a permis de discuter et parfois d'infirmer un certain nombre d'affirmations très médiatiques concernant, par exemple, les émissions de GES ou la consommation d'eau pour la production de viande. Il reste patent que la viande de bœuf est à l'origine d'impacts environnementaux plus importants que ceux d'autres viandes et plus importants par $\mathrm{kg}$ de produit que le lait. C'est la longue phase d'élevage du jeune qui est la plus préjudiciable sur la plupart des impacts environnementaux abordés ramenés au $\mathrm{kg}$ de viande produit et, à ce titre, la vache allaitante est souvent attaquée. Mais une vision holistique nuance largement ce résultat; un bilan entre les aspects positifs et négatifs de l'élevage de bovins viande en termes de production de protéines et d'environnement est proposé au tableau 3. On ne peut plus en effet se contenter d'une analyse séparant la phase d'élevage de celle de l'engraissement car c'est le même animal qui transite d'un système à l'autre. Par ailleurs, cette analyse a clairement montré que l'intensité de l'impact est très différente lorsqu'on considère les impacts par unité de surface, lorsqu'on prend en compte le stockage de carbone des prairies et enfin lorsqu'on intègre les impacts positifs des systèmes herbagers sur la biodiversité et sur le paysage. En outre, au-delà des impacts quantifiés décrits dans cet article, l'élevage de bovins viande joue un rôle culturel et patrimonial très important liés aux pratiques pastorales, aux savoir-faire, aux valeurs identitaires des paysages d'élevage et aux pratiques

Tableau 3. Bilan des avantages et des inconvénients pour l'alimentation humaine et l'environnement de la filière bovins viande par rapport aux autres filières agricoles, animales et végétales. ${ }^{(1)}$

\begin{tabular}{|c|c|}
\hline Avantages & Inconvénients \\
\hline $\begin{array}{l}\text { Utilisation maximale d'herbe pâturée et stockage } \\
\text { de carbone } \\
\text { Valorisation de terres non arables permettant } \\
\text { le maintien de la population dans les territoires } \\
\text { Maintien d'une biodiversité végétale et animale } \\
\text { Paysages ouverts et attractifs } \\
\text { Faible utilisation de pesticides } \\
\text { Impacts environnementaux modérés par ha }\end{array}$ & $\begin{array}{l}\text { Impacts élevés sur le réchauffement climatique, } \\
\text { l'eutrophisation des eaux et l'acidification des sols } \\
\text { par kg de produit } \\
\text { Impact élevé sur la perte de ressources naturelles } \\
\text { (essentiellement énergie) par kg de produit } \\
\text { Faible production d'aliments pour l'homme à l'ha }\end{array}$ \\
\hline
\end{tabular}

(1) Ce bilan très global se veut une tentative de synthèse des principaux éléments discutés dans le texte. Toutefois il est évident que la comparaison est difficile entre la filière bovin viande d'une part et des filières animales ou végétales très diverses d'autre part, et qu'avantages et inconvénients dépendent de la situation géographique et de la structure des exploitations, du niveau d'intensification et des pratiques intra-filière. 
alimentaires patrimoniales issus de la viande.

Il n'en reste pas moins que les systèmes allaitants français sont encore engagés aujourd'hui dans un processus d'agrandissement de leur surface au détriment de leur durabilité : leur efficience technique a plutôt diminué depuis 20 ans, leurs performances environnementales ne se sont pas améliorées et le revenu par travailleur s'est juste maintenu grâce aux aides publiques et aux gains de productivité du travail. Pourtant, ces systèmes de bovins viande semblent avoir beaucoup d'atouts en main pour aborder la transition agroécologique. Un système de production basé en effet sur une pro- portion importante de prairies permanentes permet un recours accru à des régulations biologiques, une dépendance moins grande aux intrants et est plus lié à son environnement physique. Dans cette optique, il faudra que ces systèmes considèrent davantage la biodiversité comme une ressource et cherchent à la préserver.

Enfin, la question du développement, ou non, de systèmes mixtes lait-viande au détriment à la fois des systèmes allaitants classiques et des systèmes laitiers très spécialisés est une question de fond à laquelle nous serons confrontés d'autant plus que la demande en viande diminue dans un pays comme la France. L'utilisa- tion de vaches laitières à potentiel laitier plus faible qu'actuellement et à bonnes qualités bouchères serait une option permettant d'assurer un revenu à l'éleveur tout en répartissant les impacts environnementaux entre les deux produits, lait et viande. Mais l'analyse environnementale de ces systèmes reste à affiner, ainsi que l'ensemble des conséquences à court et long terme de ces systèmes plus consommateurs en intrants.

\section{Remerciements}

Les auteurs sont redevables à Jacques Agabriel de ses commentaires et de sa correction pertinente de ce texte.

\section{Références}

Adler P.B., Raff D.A., Lauenroth W.K., 2001. The effect of grazing on the spatial heterogeneity of vegetation. Oecologia, 128, 465-479.

Aubertot J.N., Barbier J.M., Carpentier A., Gril J.J., Guichard L., Lucas P., Savary S., Savini I., Voltz M., 2005. Pesticides, agriculture et environnement. Réduire l'utilisation des pesticides et en limiter les impacts environnementaux. Rapport d'expertise collective, INRA, Cemagref, 902p.

Aubin J., 2014. Empreinte environnementale de la viande et des produits carnés. $15^{\text {emes }}$ Journées Sciences du Muscle et Technologies des Viandes, Clermont-Ferrand, Viandes Produits Carnés, VPC-2014-30-6-2, 7p.,

www.viandeetproduitscarnes.com

Benton T.G., Bryant D.M., Cole L., Crick H.Q.P., 2002. Linking agricultural practice to insect and bird populations: a historical study over three decades. J. Appl. Ecol., 39, 673-687.

Benton T.G., Vickery J.A., Wilson J.D., 2003 Farmland biodiversity: is habitat heterogeneity the key? Trends Ecol. Evol., 18, 182-188.

Berton M., Lherm M., Agabriel J., Gallo L., Ramanzin M., Sturaro E., 2016. Assessing the sustainability of a combined extensive/intensive beef production system: the case of French suckler cow-calf farms integrated with Italian beef fattening herds. Opt. Medit., 116, 57-61.

Castillon P., 2005. Le phosphore : sources, flux et rôles pour la production végétale et l'eutrophisation. In : Numéro spécial, Le phosphore dans l'alimentation animale. Meschy F., Sauvant D., Pinot P. (Eds). INRA Prod. Anim., 18, 153-158.

Charroin T., Galan F., Capitain M., 2006. Les consommations d'énergie dans les systèmes d'élevage bovin. Première contribution des Réseaux d'Élevage. Fourrages, 186, 179-191.

Citepa, 2016. Rapport national d'inventaire pour la France au titre de la convention cadre des Nations Unies sur les changements climatiques et du protocole de Kyoto. Citepa, Paris, France, $527 \mathrm{p}$.

Corson M.S., Doreau M., 2013. Évaluation de l'utilisation de l'eau en élevage. INRA Prod. Anim., 26, 239-248.

Couvreur J.P., 2006. Quelques leviers pour maîtriser la consommation d'énergie et optimiser la mécanisation de 1'exploitation. Fourrages, 187, 201-310.

Cruz P., Duru M., Thérond O., Theau J.P., Ducourtieux C., Jouany C., Al Haj Khaled R., Ansquer P., 2002. Une nouvelle approche pour caractériser les prairies naturelles et leur valeur d'usage. Fourrages, 172, 335-354.

De Boer I.J.M., 2003. Environmental impacts assessment of conventional and organic milk production. Livest. Prod. Sci., 80, 69-77.

Dengler J. Janisova M., Torok P., Wellstein C., 2014. Biodiversity of Palaearctic grasslands: a synthesis. Agricult. Ecosys. Environ., 82, 1-14.

De Vries M., Van Middelaar C.E., De Boer I.J.M., 2015. Comparing environmental impacts of beef production systems: a review of life cycle assessments. Livest. Sci., 178, 279-288.

Devun J., Agabriel J., Moreau S., Manneville V., 2016. Quels sont les liens entre les pratiques d'élevage et les bilans production - environnement? L'exemple de systèmes charolais. Fourrages, 228, 283-293.

Dollé J.B., Agabriel J., Peyraud J.L., Faverdin P., Manneville V., Raison C., Gac A., Le Gall A., 2011. Les gaz à effet de serre en élevage bovin : évaluation et leviers d'action. In : Gaz à effet de serre en élevage bovin : le méthane. Doreau M., Baumont R., Perez J.M. (Eds). Dossier, INRA Prod. Anim., 24, 415-432.

Doreau M., Corson M.S., 2017. Production de viande et ressource en eau. Viande Produits Carnés, VPC-2017-33-2-1, 8p.,

www.viande-etproduitscarnes.com

Doreau M., van der Werf H.M.G., Micol D., Dubroeucq H., Agabriel J., Rochette Y., Martin C., 2011. Enteric methane production and greenhouse gases balance of diets differing in concentrate in the fattening phase of a beef production system. J. Anim. Sci., 89, 2518-2528.

Doreau M., Martin C., Morgavi D.P., 2017. Réduire les émissions de méthane entérique par l'alimentation des ruminants. Viande Produits Carnés, sous presse.

www.viandeetproduitscarnes.com

Dumont B., Farruggia A., Garel J.P., 2007. Pâturage et biodiversité des prairies permanentes. Renc. Rech. Rum., 14, 17-24.
Dumont B., Farruggia A., Garel JP, Bachelard P., Boitier E., Frain M., 2009. How does grazing intensity influence the diversity of plants and insects in a species-rich upland grassland on basalt soils? Grass Forage Sci., 64, 92-105.

Dumont B., Carrère P., Ginane C., Farruggia A., Lanore L., Tardif A., Decuq F., Darsonville O., Louault F., 2011. Plant-herbivore interactions affect the initial direction of community changes in an ecosystem manipulation experiment. Basic Appl. Ecol., 12, 187-194.

Dumont B., Dupraz P., Aubin J., Benoit M., Bouamra-Mechemache Z., Chatellier V., Delaby L., Delfosse C. Dourmad J.Y., Duru M., Frappier L., Friant-Perrot M., Gaigné C., Girard A., Guichet J.L., Havlik P., Hostiou N., Huguenin-Elie O., Klumpp K., Langlais A., Lemauviel-Lavenant S., Le Perchec S., Lepiller O., Méda B., Ryschawy J., Sabatier R., Veissier I., Verrier E., Vollet D., Savini I., Hercule J., Donnars C., 2016, Rôles, impacts et services issus des élevages en Europe. Synthèse de l'expertise scientifique collective, INRA, France, $133 \mathrm{p}$.

Farruggia A., Dumont B., Jouven M., Baumont B., Loiseau P., 2006. La diversité végétale à l'échelle de l'exploitation en fonction du chargement dans un système bovin allaitant du Massif central. Fourrages, 188, 477-493.

Farruggia A., Martin B., Baumont R., Prache S., Doreau M., Hoste H., Durand D., 2008. Quels intérêts de la diversité floristiques des prairies permanentes pour les ruminants et les produits animaux, INRA Prod. Anim., 21, 181-199.

Farruggia A., Dumont B., Scohier A., Leroy T., Pradel P., Garel J.P., 2012. An alternative rotational stocking management designed to favour butterflies in permanent grasslands. Grass Forage Sci., 67, 136-149.

Gac A., Ledgard S., Lorinquer E., Boyes M., Le Gall A., 2012. Carbon footprint of sheep farms in France and New Zealand: comparison of methods and methodology analysis. In: Corson M.S., Van der Werf H.M.G. (Eds). $8^{\text {th }}$ Int. Conf. LCA Food, Saint-Malo, p50.

Gac A., Béchu T., 2014. L'empreinte eau consommative du lait et de la viande bovine et ovine: premiers repères sur des systèmes français. Renc. Rech. Rum., 21, 39-42. 
Gerber P.J., Steinfeld H., Henderson B., Mottet A., Opio C., Dijkman J., Falcucci A., Tempio G., 2013. Tackling climate change through livestock - A global assessment of emissions and mitigation opportunities. FAO, Rome, Italy.

Gerber P.J., Mottet A., Opio CI., Falcucci A., Teillard F., 2015. Environmental impacts of beef production: Review of challenges and perspectives for durability. Meat Sci., 109, 2-12.

Giec, 2007. Bilan 2007 des changements climatiques. Contribution des Groupes de travail I, II et III au quatrième rapport d'évaluation du groupe d'experts intergouvernemental sur l'évolution du climat. Giec, Genève, Suisse, 103p.

Hoekstra A.Y., Chapagain A.K., Aldaya M.M., Mekonnen M.M., 2011. The water footprint assessment manual: setting the global standard. Earthscan Publishing, Londres, Royaume-Uni.

Institut de l'Élevage, 2014. Guide de l'alimentation du troupeau bovin allaitant. Vaches, veaux et génisses de renouvellement. Agabriel J., Brouard S., Devun J. (Eds). Institut de l'Élevage, Paris, France, 340p.

Kanyarushoki C., Defrance P., Farruggia A., Monsallier F., Loisel A., Hulin S., Jourjon F., 2015. Evaluation de la performance environnementale (ACV) des exploitations laitières en polyculture-élevage : base pour un changement de pratiques au sein des systèmes de production ? Renc. Rech. Rum., 22, 75

Klumpp K., Guix N., Tallec T., Soussana J.F., 2010. La pratique agricole a-t-elle plus d'impact que la variabilité climatique sur le stockage du C en prairie pâturée ? Renc. Rech. Rum., 17, 343-346.

Klumpp K., Faverdin P., Benoit M., Pellerin S., Bamière L., Chemineau P., Lherm M., Pardon L., Doreau M., 2013. Réduction des émissions de gaz à effet de serre des ruminants : choix des techniques les plus efficaces, ampleur de la réduction, coût de leur mise en application. Renc. Rech. Rum., 20, 315-318

Larue A., Moreau S., Agabriel J., Devun J., Farrié J.P., Renon J., Brunschwig G., Manneville V., 2012. Bilan production - environnement de deux systèmes bovins allaitants contrastés. Renc. Rech. Rum., 19, 60

Lherm M., Agabriel J., Devun J., Etat des lieux et évolutions de la production bovine allaitante en France et dans trois pays européens. In Elevage bovin allaitant. Agabriel J., Renand G., Baumont R. (Eds). Dossier, INRA Prod. Anim., 30, 93-106.

Luka H., Daniel C., Barloggio G, Pfiffner L., 2016. Les bandes fleuries régulent les ravageurs des cultures maraichères et favorisent la biodiversité. Recherche Agronomique Suisse, 7, 268275 .

Luoto M., Pykälä J., Kuussaari M., 2003. Decline of landscape-scale habitat and species diversity after the end of cattle grazing. J. Nat. Conserv., 11, 171-178.

MacDonald D., Crabtree J.R., Wiesinger G., Dax T., Stamou N., Fleury P., Gutierrez J.L., Gibon A., 2000. Agricultural abandonment in mountain areas of Europe: environmental consequences and policy response. J. Environ. Manag., 59, 47-69.

McKinsey \& Company, 2009. Pathways towards a low-carbon economy. Version 2 of the global greenhouse gas abatement cost curve, McKinsey \& Co, USA.

Martin E., Mathias E., 2013. Analyse du potentie de 10 actions de réduction des émissions d'ammoniac des élevages français aux horizons 2020 et 2030. Rapport, Ed. Ademe, Angers, France, $242 \mathrm{p}$

Mekonnen M.M., Hoekstra A.Y., 2010. The green, blue and grey water footprint of farm animals and animal products. Volume 1: main report. Value of water research report no 48 Unesco-IHE Institute of Water education, Delft, Pays-Bas, 45p

Metson G.S., Cordell D., Ridoutt B., 2016 Potential impact of dietary choices on phosphorus recycling and global phosphorus footprints the case of the average Australian city. Front Nutr., 3, article 36, doi: 10.3389/fnut.2016.00035.

Moran D., MacLeod M., Wall E., Eory V., McVittie A., Barnes A., Rees R.M., Topp C.F.E. Pajot G., Matthews R., Smith P., Moxey A. 2011. Developing carbon budgets for UK agriculture, land-use, land-use change and forestry out to 2022. Climatic Change, 105, 529-553.

Moreau S., Manneville V., Morel K., Agabrie J., Devun J., 2013. Le compromis performances de production et impacts environnementaux méthode et analyse des résultats dans les élevages bovins allaitants. Renc. Rech. Rum., 20 311-314.

Morel K., Farrié J.P., Renon J., Manneville V. Agabriel J., Devun J., 2016, Environmental impacts of cow-calf beef systems with contrasted grassland management and animal production strategies in the Massif central, France. Agricult. Sys., 144, 133-143.

Nguyen T.T.H., van der Werf H.M.G., Doreau M., 2012a. Life cycle assessment of three bullfattening systems: effect of impact category on ranking. J. Agric. Sci., 150, 755-763.

Nguyen T.T.H., van der Werf H.M.G., Eugène M., Veysset P., Devun J., Chesneau G., Doreau M., 2012b. Effect of type of ration and allocation methods on the environmental impacts of beef-production systems. Livest. Sci., 145 239-251.

Nguyen T.T.H., Doreau M., Eugène M., Corson M.S., Garcia-Launay F., Chesneau G. van der Werf H.M.G., 2013a. Effect of farming practices for greenhouse gas mitigation and subsequent alternative land-use on environmental impacts of beef-cattle production systems Animal, 7, 860-869

Nguyen T.T.H., Doreau M., Corson M.S. Eugène M., Delaby L., Chesneau G., Gallard Y., Van der Werf H.M.G., 2013b. Effect of dairy production system, breed and co-product handling methods on environmental impacts at farm level. J. Environ. Manag., 120, 127-137.

Norderhaug A., Ihse M., Pedersen O., 2000 Biotope patterns and abundance of meadow plant species in a Norwegian rural landscape. Landscape Ecol., 15, 201-218.

Ogino A., Orito H., Shimada K., Hirooka H., 2007. Evaluating environmental imapcts of the Japanese beef cow-calf system by the life cycle assessment method. Anim. Sci. J., 78, 424-432.

Orlandi S., Probo M., Sitzia T., Trentanovi G., Garbarino M., Lombardi G., Lonati M., 2016. Environmental and land use determinants of grassland patch diversity in the western and eastern Alps under agro-pastoral abandonment. Biodivers. Conserv., 25, 275-293

Pellerin S., Bamière L., Angers D., Béline F., Benoit M., Butault J.P., Chenu C., ColnenneDavid C., De Cara S., Delame N., Doreau M., Dupraz P., Faverdin P., Garcia-Launay F. Hassouna M., Hénault C., Jeuffroy M.H., Klumpp K., Metay A., Moran D., Recous S., Samson E., Savini I., Pardon L., 2015. Agriculture et gaz à effet de serre. Dix actions pour réduire les émissions. Ed. Quae, Versailles, France, 198p.

Pöyry J., Luoto M., Paukkunen J., Pykälä J., Raatikainen K., Kuussaari M., 2006. Different responses of plants and herbivore insects to a gradient of vegetation height: an indicator of the vertebrate grazing intensity and successional age. Oikos, 115, 401-412

Pykälä J., 2003. Effects of restoration with cattle grazing on plant species composition and richness of semi-natural grasslands. Biodivers. Conserv., 12, 2211-2226.

Réseau d'Information Comptable Agricole, 2014 http://agreste.agriculture.gouv.fr/enquetes/rese au-d-information-comptable

Ridoutt B.G., Pfister S., 2013. A new water footprint calculation method integrating consumptive and degradative water use into a single stand-alone weighted indicator. Int. J. Life Cycle Assess., 18, 204-207.

Ridoutt B.G., Sanguansri P., Freer M., Harper G.S., 2012. Water footprint of livestock: comparison of six geographically defined beef production systems. Int. J. Life Cycle Assess. $17,165-175$

Salou T., Le Mouël C., Van der Werf H.M.G., 2017. Environmental impacts of dairy system intensification: the functional unit matters! J Cleaner Prod., 140, 445-454.

Schulte R.P.O., Lanigan G., Gibson M., 2011. Irish agriculture, greenhouse gas emissions and climate change: opportunities, obstacles and proposed solutions. Ed. Teagasc, Oak Park, Irlande.

Soussana J.F., Klumpp K., Ehrhardt F., 2014. The role of grassland in mitigating climate change. Grassland Sci. Europe, 19, 75-87.

Steinfeld H., Gerber P., Wassenaar T., Castel V., Rosales M., De Haan C., 2006. Livestock's long shadow.: environmental issues and options. FAO, Rome, Italy, 390p.

Tschumi M., Albrecht M., Bärtschi C., Collatz J., Entling M.H., Jacot K., 2016. Perennial, species-rich wildflower strip enhance pest control and crop yield. Agric. Ecosys. Environ. 220, 97-103

Veysset P., Lherm M., Bébin D., 2011. Productive, environmental andd economic performances assessments of organic and conventional suckler cattle farming systems. Org. Agric., 1, 1-16.

Veysset P., Lherm M., Roulenc M., Troquier C., Bébin D., 2015. Productivity and technical efficiency of suckler beef production systems: trends for the period 1990 to 2012. Animal, 9, 2050-2059.

Veysset P., Mosnier C., Lherm M., 2016. Beef cattle farms in less-favoured areas: drivers of 
sustainability over the last 24 years. Implications for the future. Opt. Médit. - Mountain pastures and livestock farming facing uncertainty: environmental technical and socio economic challenges, A 116, 27-38.

Vickery J.A., Tallowin J.R., Feber R.E., Asteraki E.J., Atkinson P.W., Fuller R.J., Brown V.K.,
2001. The management of lowland neutral grasslands in Britain: effects of agricultural practices on birds and their food resources. J. Appl. Ecol., 38, 647-664.

Wallis De Vries M.F., Parkinson A.E., Dulphy J.P., Sayer M., Diana E., 2007. Effects of livestock breed and grazing intensity on biodiversity and production in grazing systems. 4. Effects on animal diversity. Grass Forage Sci., 62, 185-197. Williams A.G., Audsle, E., Sandars D.L., 2006. Determining the environmental burdens and resource use in the production of agricultural and horticultural commodities. Main Report. Defra Research Project IS0205. Bedford: Cranfield University et Defra.

\title{
Résumé
}

L'ambition de cet article est de dresser un bilan environnemental de l'élevage de bovins pour la viande en France, aussi exhaustif que possible, à partir des données scientifiques et statistiques disponibles. Les impacts environnementaux abordés sont les émissions de gaz à effet de serre, la pollution de l'eau et des sols, l'utilisation des ressources naturelles (énergie, phosphore, eau), l'utilisation des terres et la biodiversité végétale et animale. Les méthodologies d'évaluation et les indicateurs utilisés sont justifiés et discutés. Lorsque l'unité d'expression est le kg de viande, l'essentiel des impacts environnementaux provient de l'atelier naisseur en raison de la longue phase d'élevage pour obtenir un broutard. Les résultats sont très différents lorsque les impacts sont calculés par unité de surface, que le stockage de carbone dans les prairies est pris en compte et que les impacts positifs de ces systèmes sur la biodiversité et sur le paysage sont intégrés à l'analyse. Bien qu'ayant des atouts pour aborder la transition agroécologique du fait de la part importante de prairies permanentes leur permettant de tirer avantage des régulations biologiques, les systèmes naisseurs sont cependant engagés depuis 20 ans dans un processus d'agrandissement au détriment de leur durabilité.

\begin{abstract}
Environmental amenities and impact of French farms for beef production

This paper is aimed at providing a comprehensive review of environmental issues related to beef cattle farming in France, including available scientific knowledge and statistics. Greenhouse gas emissions, water and soil pollution, use of natural resources (energy, phosphorus, water), land use, and animal and plant biodiversity are addressed. Methodology and indicators for evaluation are presented and discussed. When the results were expressed as kg beef, most impacts were due to suckling cow farms (calf-to-weanling) due to the long breeding time necessary to produce weanlings. The results were, however, very different when they were expressed per hectare, when carbon storage by soil was taken into account and when the positive impacts of these systems on biodiversity were included in the analysis. Despite a certain advantage for making a transition towards agroecology due to the large part of permanent grasslands, weanling cow farms have been evolving for 20 years towards the expansion of their structures at the expense of their sustainability.
\end{abstract}

DOREAU M., FARRUGGIA A., VEYSSET P., 2017. Aménités et impacts sur l'environnement des exploitations françaises élevant des bovins pour la viande. In : Élevage bovin allaitant. Agabriel J., Renand G., Baumont R. (Eds). Dossier, INRA Prod. Anim., 30, 165-178. 
